\title{
Inhibiting STAT3 and Activating TLR Against Tumors with OPSS Liposomes, Curcumin, and TLR Agonists
}

\author{
Shreya Sujith ${ }^{1}$, Vani Pillai ${ }^{1}$, Keaton Rasic ${ }^{2}$, Marisabel Ramirez ${ }^{3}$, Max Kullberg ${ }^{3}$, and \\ Holly Martinson ${ }^{3}$
}

${ }^{1}$ Robert Service High School, Anchorage, AK, USA

${ }^{2}$ University of Washington, Seattle, WA, USA

${ }^{3}$ University of Alaska, Anchorage, AK, USA

\section{$\underline{\text { ABSTRACT }}$}

In solid malignancies such as breast cancer, the tumor cells create an immunosuppressive environment through the aid of macrophages. The macrophages become tumor-associated and inhibit a T-cell response critical to removing the cancerous cells. The main signaling pathway responsible for the macrophages' pro-tumoral behavior is the STAT3 signaling pathway, a subset of the JAK/STAT signaling pathway that prevents the toll-like receptor (TLR) expression. TLRs are essential to the immune system's anti-tumoral response. Therefore, to repolarize the macrophages in from a pro-tumoral to an anti-tumoral form, curcumin (STAT3 inhibitor), and TLR agonists (TLR stimulant) were delivered to a co-culture using complement C3 liposomes (OPSS liposomes). The OPSS liposomes cause macrophages to uptake the liposomes, allowing for targeted drug delivery to macrophages. 4T1 cancer cells and bone marrow-derived monocytes were co-cultured and treated with control liposomes containing PBS, OPSS liposomes containing curcumin and TLR agonist, and OPSS liposomes containing TLR agonist. RT-qPCR measured gene expression, and flow cytometry measured protein expression. This study's results point to the effectiveness of the OPSS liposome, curcumin, and TLR agonist treatment. The combination of curcumin and TLR agonist appear to effectively inhibit the STAT3 signaling pathway and activate the expression of TLR. Future studies should implement the OPSS liposome, curcumin, and TLR agonist treatment in a mouse model and observe for a potential T-cell response.

\section{Introduction}

Around the world, everyone in six deaths is due to cancer, making it the second leading cause of death, according to the Global Health Metrics (2017). As stated by the Centers for Disease Control and Prevention, between 2010 and 2020 , the prevalence of cancer increased by over $24 \%$ in men and $21 \%$ in women (2018). The National Cancer Institute defines cancer as the uncontrolled growth of mutated cells in the body that do not die and form a mass of abnormal tissue called a tumor (2017). There are two main divisions of cancer: solid tumor cancers and blood cancers. Solid tumor cancers, also known as solid malignancies, do not contain cysts or liquid, while blood cancers form in the bloodstream and are not solid masses of cancer cells (National Cancer Institute 2017). In this study, the main focus was the solid malignancies, which, according to the Leukemia and Lymphoma Society, account for approximately 90\% of new cancer cases diagnosed in 2019 (2019).

In solid malignancies, there is a considerable amount of macrophages present in the tumor microenvironments, which was further discussed in a study done by Dr. Sousa and colleagues at the University of Helsinki (2015). Macrophages are immune cells essential to maintaining tissue health within the body because they detect and destroy bacteria along with other harmful substances. However, in the tumor microenvironment, macrophages help contribute to the tumor's immunosuppressive environment, thereby becoming tumor-associated macrophages that inhibit a Tcell response. T-cell responses are critical aspects of the immune response, and their suppression can lead to metastasis 
of tumors. These macrophages suppress not just the immune response against the tumor, but also the current treatments against cancer, such as chemotherapy, radiation therapy, and immunotherapy (Sousa et al., 2019).

According to Dr. DeNardo of the Washington University in St. Louis and Dr. Ruffell of the H. Lee Moffitt Cancer Center, there are no feasible therapies that target the immunosuppressive macrophages (2019). Due to the critical role that macrophages play in contributing to the tumor's immunosuppressive environment, recent research has focused upon creating a targetable therapy to inhibit the association of the macrophages with the tumor.

\section{Literature Review}

\section{Curcumin}

Numerous studies have been conducted to determine a plausible therapy to inhibit the association of macrophages with tumors. One of the more promising compounds that have been extensively researched is curcumin, a compound found in the turmeric root with anti-inflammatory and anti-tumoral properties (Kunnumakkara et al., 2019). Dr. Ajaikumar Kunnumakkara and colleagues at the Department of Biosciences and Bioengineering in the Indian Institute of Technology have studied extensively the positive effects of curcumin on multiple diseases, most notably cancer. In a research review, Dr. Kunnumakkara further affirms the significance of the therapeutic effects of curcumin on cancer. The review analyzed the results of several studies and concluded that curcumin interacts with cell signaling pathways in cancer cells and inhibits several of the growth mechanisms of tumors (2017). In a contrasting perspective, Dr. Patrick and Dr. Stanbrook of the University of Toronto Department of Medicine raised the question of the effectiveness of curcumin as a possible medical treatment (2018). In their research review, Dr. Patrick and Dr. Stanbrook attempted to undermine the effects of curcumin in medical treatment. However, the review only focused on a single study that indicated the ineffectiveness of curcumin as a cancer treatment. They failed to consider multiple other studies with statistically significant results, such as the those that Dr. Kunnumakkara and colleagues had considered.

According to a study by Dr. Zhang and colleagues of the Chongqing Medical University, tumors can "secrete factors" that polarize macrophages and cause them to undergo a change beneficial to the tumor microenvironment (2013). One particular study done by Dr. Varalakshmi and colleagues at the Centre for Cellular and Molecular Biology in Hyderabad, India, helped to affirm that the administration of curcumin to the tumor can help repolarize the macrophages into a form that that is more anti-tumoral. In their study, it was found that when curcumin was administered to tumor-associated macrophages in-vitro, the tumor cells were forced to undergo apoptosis, also known as programmed cell death. Additionally, the macrophages were not polarized into becoming tumor-associated (2008). Although this study is a bit outdated, the results were consistent with recent research. A clear example is shown in a study done by Dr. Mukherjee and colleagues at the City University of New York Graduate Center, which further affirms the role of curcumin and the repolarization of macrophages in a tumor microenvironment. The results of this study showed that curcumin had a direct effect on eliminating cancer cells, while it repolarized the tumor-associated macrophages into becoming the non-tumor-associated form (2016). While the study done by Dr. Varalakshmi focused on the overall effect of curcumin on solid malignancies, the study done by Dr. Mukherjee offered a more focused perspective on the impact of curcumin on tumor-associated macrophages in glioblastoma, also known as brain cancer.

\section{TLRs and TLR agonists}

To fully understand the effects of curcumin on the tumor-associated macrophages, it is essential to recognize the main signaling mechanism that influences the immunosuppressive environment of the tumor. According to Delneste et al. of the University Hospital of Angers, toll-like receptors (TLRs) are a class of proteins that line the membranes of all cells, including the tumor. In macrophages, they help detect molecules from invading pathogens and stimulate an immune response (2007). TLRs do this by binding to molecules characteristic of specific pathogens (Delneste et al., 
2007). If TLRs can be activated in a tumor microenvironment, the immune cells can be stimulated to attack the tumor as if it were attacking a pathogen. A recent study done by Dr. Feng et al. at the University of Macau and Nanjing University investigated the potential methods and advantages of switching macrophages from being pro-tumoral to anti-tumoral by controlling the TLR signaling pathways (2019). This study introduced the idea of using agonistssubstances that initiate a physiological response when binding to a receptor (such as TLRs) — to generate macrophages with anti-tumoral properties. The results are significant because they express the potential advantages of controlling the TLR signaling pathways. In a research review by Dr. Boozari, Dr. Butler, and Dr. Sahebkar of Mashhad University of Medical Sciences, the potential use of curcumin to modulate the signaling pathways of TLR was examined (2018). In terms of cancer, they found that curcumin helped stimulate the immune system by controlling the TLR signaling pathways. This conclusion suggests that curcumin and TLR agonists can modulate the TLR signaling pathways in macrophages, thereby repolarizing the tumor-associated macrophages and making them anti-tumoral.

\section{STAT3 Pathway}

One of the main signaling pathways that could influence macrophage repolarization is the Janus kinase/signal transducers and activators of transcription (JAK/STAT) pathway. In this study, the main focus was a subtype of the JAK/STAT pathway, which is prevalent in solid malignancies, known as the STAT3 pathway. The STAT3 pathway supports tumor growth, survival, immunosuppression, and metastasis (Huynh et al., 2019). Targeting the receptors of STAT3 is appearing beneficial in curbing the growth of cancerous cells and increasing anti-tumor immunity. According to Dr. Johnson and colleagues of the University of California, San Francisco, treatments that target the STAT3 signaling pathway can provide therapeutic benefit by inhibiting tumor growth and the stimulation of anti-tumoral immunity (2018). By targeting and inhibiting the STAT3 pathway using a STAT3 inhibitor such as curcumin, there could be beneficial results in decreasing tumor growth, increasing overall anti-tumor immunity, and allowing for the expression and activation of the TLRs. Concerning curcumin and the STAT3 pathway, another research review by Dr. Kunnumakkara and colleagues revealed that curcumin inhibited the STAT3 pathway, thereby inhibiting tumor growth in ovarian cancer, pancreatic cancer, and multiple myeloma (2017). Through the conclusions of this review, the potential therapeutic effects of curcumin and TLR agonists become evident.

\section{OPSS Liposomes}

In the discussion of curcumin and TLR agonists and their potential benefits in inhibiting tumor growth, it is also essential to discuss a significant gap in the current field of immuno-oncology. Despite the research about the mechanisms of curcumin and TLR agonists and their effects on tumors, delivery mechanisms such as liposomes must be explored extensively as potential treatments for cancer (Kunnumakkara et al., 2017). As defined by Dr. Akbarzadeh and colleagues, liposomes are sphere-shaped vesicles, or sacs, that can be utilized as a drug delivery mechanism (2013). Given the preceding context, curcumin and TLR agonists could be delivered to the tumor microenvironment using liposomes. In a study conducted by Dr. Kullberg and colleagues at the University of Alaska Anchorage, suppressor cells, such as macrophages, were targeted using a type of liposome called complement C3 liposomes (2015). As defined by the University of Rochester Health Center Encyclopedia, complement C3 is a protein found in the blood that binds to pathogens for the immune system to target and destroy (2019). The complement C3 liposomes (referred to as OPSS liposomes) cause macrophages to uptake the liposomes, which allows for targeted drug delivery to macrophages. In a separate study by Dr. Kullberg and colleagues, there was increased uptake of OPSS liposomes by macrophages (2019). The results of this study are significant, by the fact that this is a viable drug delivery mechanism that can be specific to macrophages. Delivering curcumin and TLR agonists via OPSS liposomes to tumor-associated macrophages could serve as a targetable therapy to the immunosuppressive macrophages, thereby reprogramming and activating them to create an immune response against a tumor. 


\section{Methods}

In this study, the efficacy of the curcumin and TLR agonist delivery mechanism to macrophages using OPSS liposomes was evaluated using an in vitro breast cancer co-culture model. The co-culture model was utilized to simulate a tumor microenvironment that contained both macrophages and tumor cells. The effect of the OPSS liposome, curcumin, and TLR agonist treatment was determined by measuring immune stimulatory factors on bone marrow-derived myeloid cells (BMDMs) and mouse mammary carcinoma 4T1 tumor cells by analyzing protein and gene expression using qualitative Reverse Transcription Polymerase Chain Reaction (RT-PCR) and flow cytometry.

\section{Treatments}

The treatments delivered using C3-liposomes to BMDMs in a breast cancer tumor co-culture model included phosphate buffer saline (PBS), TLR agonists, and a combination of TLR agonists and curcumin. Curcumin and TLR agonists both target TLRs, which can both be utilized to stimulate an immune response against tumors. OPSS liposomes were used due to the results described in a study conducted by Dr. Kullberg et al. (2019). The study showed increased uptake of OPSS liposomes by myeloid cells compared to control liposomes in mice.

Curcumin was used due to the immunostimulatory effects described by Dr. Mukherjee et al. in a study investigating the effect of curcumin on macrophages in glioblastoma (2016).

Although this particular study delivered the curcumin intranasally into live mice, the results demonstrate that curcumin causes macrophages to repolarize into an anti-tumoral form, thereby stimulating an immune response against the tumor. TLR agonists were utilized due to their ability to stimulate TLRs, resulting in an anti-tumor response outlined in a study performed by Dr. Michaelis et al. of the Oregon Health and Science University (2019). The TLR agonist used in this experiment was R848, a TLR7/8 agonist. A study conducted by Dr. Michawlis et al. employed R848 and found that R848 enhanced the immune response against the tumor (2019).

\section{Preparation of Liposomes}

To measure the effect of curcumin delivery using the OPSS liposomes, the liposomes prepared in this category included control liposomes containing phosphate-buffer saline (PBS), OPSS liposomes containing TLR agonist, and OPSS liposomes containing curcumin and TLR agonist. Due to the aforementioned immunostimulatory effects of both curcumin and TLR agonists, the results of a combination of these two compounds were examined using OPSS liposomes containing curcumin and TLR agonists.

Using the methods outlined in Complement C3 mediated targeting of liposomes to granulocytic myeloidderived suppressor cells by Dr. Kullberg et al., OPSS liposomes were prepared (2015). All the liposomes were developed using a thin-film hydration method followed by extrusion. This method involves removing an organic solvent, chloroform, which leads to the creation of a thin lipid film. The control liposomes contained four different formulations of lipids (DPPC, DSPC, DSPE-PEG (2000), and DSPE-PEG(2000)), while the OPSS liposomes contained the same lipids, except the lipid DSPE-PEG(2000) was substituted for DSPE-PEG(2000)-PDP. To produce OPSS liposomes with curcumin, the 4 lipids, rhodamine dye, and curcumin were mixed in chloroform in an 83:12:1:3:1:1 ratio. The lipid mixtures were dried under a nitrogen stream for 1 hour. The liposomes which acted as a control for the experiment were rehydrated with $0.7 \mathrm{~mL}$ of filtered water. For liposomes containing TLR agonists, the compounds were added to water before rehydration. After the dispersion medium was added and agitated, the liposomes were obtained through extrusion.

The extruded liposomes were then purified using the methods outlined by Dr. Kullberg et al. (2019). The liposomes were purified using a CL-4B sepharose column hydrated in phosphate-buffer saline, an agarose gel filtration base. The rhodamine dye was used to measure the lipid concentration in the liposomes. Using a spectrophotometer 
NanoDrop 2000 by ThermoFisher Scientific, the purity and concentration of the lipids were assessed. The rhodamine peak was observed to dilute the lipid concentration to $0.875 \mathrm{mg} \operatorname{lipid} / \mathrm{mL}$.

\section{Co-culture}

In order to best simulate a tumor microenvironment and the interactions between the cells, a co-culture model containing both tumor cells and BMDMs were used. A similar method was used in a study done by Dr. Zhang et al. of Chongqing Medical University. In the study, encapsulated hydrazinocurcumin (a synthetic analog of curcumin) was delivered to a co-culture containing macrophages and tumor cells using nanoparticles. As outlined by the methods of the aforementioned study, 4T1 tumor cells were cultured together with BMDMs at a 1:1 ratio of 300,000 cells in a 24well culture plate at $37^{\circ} \mathrm{C}$ and $5 \% \mathrm{CO} 2$. The different liposomes formulations were then added to separate co-culture wells and incubated for 24 hours. This process was repeated for 5 trials.

\section{RT-PCR}

Reverse Transcription Polymerase Chain Reaction (RT-PCR) is a method used to measure the RNA expression levels in cells. Following methods set by Dr. Lu et al. in their study, Effect of curcumin on human colon cancer multidrug resistance in vitro and in vivo, the process of RT-PCR was administered for each of the 5 trials conducted (2015). Following liposome treatment, $200 \mu \mathrm{L}$ TRIZOL reagent was utilized to isolate RNA from cells, following the manufacturer's instructions (Life Technologies). RNA was then resuspended in $20 \mu \mathrm{L}$ nuclease-free water, after which the concentration and purity were determined by $260 \mathrm{~nm}$ and $280 \mathrm{~nm}$ fluorescence using the Nanodrop. cDNA was synthesized from $1 \mu \mathrm{g}$ of RNA using the iScript cDNA Synthesis kit from Bio-Rad following manufacturer's instructions.

SYBR green, a green-fluorescent dye that binds to DNA, was utilized to create several copies of the cDNA produced in the previous step. Forward and reverse primers, which were necessary to copy the cDNA, were used at a concentration of $1 \mu \mathrm{L}$ and brought up to a final reaction volume of $10 \mu \mathrm{L}$ with nuclease-free water. Mouse primers were purchased from Integrated DNA Technologies and are listed in Table 1 in Appendix A.

The genes analyzed were IL-1 $\beta$, TLR 2, TLR 4, TLR 8, TLR 9, TNF- $\alpha$, IRF-7, and IP-10. As used by Dr. Mori et al. in their study, $\beta$-Actin was utilized as a reference gene to normalize gene expression for all the genes measured (2008). The expression of all the genes was measured relative to the expression of $\beta$-Actin.

\section{Flow Cytometry}

The following antibodies utilized for this study were purchased from Biolegend:

CD45-FITC, CD11b-APC, MHCII-BV510, CD40-PerCP/Cy5.5, and CD83-BV650. These antibodies were used to ascertain the percent positive expression of MHC-II, CD40, and CD83 of myeloid cells in vitro. The protein expression of the cells was measured by suspending the macrophages in phosphate-buffered saline (PBS) and stained using fluorescent tags attached to antibodies that bind to unique components of the macrophages. The samples were passed through a flow cytometer, which contains lasers and photodetectors that analyzed protein expression within and outside of the cell by detecting the aforementioned bound fluorescence antibodies.

\section{Results}

\section{RT-qPCR Results}

In order to determine the gene expression of the immune stimulatory factors by bone marrow-derived myeloid cells (BMDMs), Reverse Transcription Quantitative Polymerase Chain Reaction (RT-qPCR) was utilized. The genes 
analyzed were IL-1 $\beta$, TLR 2, TLR 4, TLR 8, and TLR 9. As used by Dr. Mori et al. in their study, $\beta$-Actin was utilized as a reference gene to normalize gene expression for all the genes measured (2008). The expression of all the genes was measured relative to the expression of $\beta$-Actin. From the 5 trials, the average relative expression of TLR $2,4,8$, and 9 with each treatment was determined and subsequently graphed in a bar graph. Data are presented as mean \pm standard error. Differences among groups were determined using an unpaired two-tailed Student's t-test. P-values of less than 0.05 were considered significant and are indicated by an asterisk over the data or a bracket between samples.

IL-1 $\beta$ and TLR form a receptor "superfamily" that recognize pathogens. All the receptors in this family have in common a toll-IL-1 receptor domain. By measuring the expression of the IL-1 $\beta$ gene, it can be assumed that the expression of this gene can also mean the expression of the TLR genes. From the 5 trials conducted, the average relative expression of IL-1 $\beta$ with each treatment was determined and subsequently graphed in a bar graph (Figure $1 \mathrm{~A}$ ). However, to be certain, the expression of the TLR 2, 4, 8, and 9 genes were also measured. Data on the gene expression of IL-1 $\beta$ and TLR 2, 4, 8, and 9 with each treatment can be found in Figures 1-2 below.

The expression of IL-1 $\beta$ has been shown to induce the expression of TNF- $\alpha$, a protein essential for resistance to infection, and cancers (Ledesma et al., 2004). TNF- $\alpha$ is responsible for necrosis and apoptosis in cancerous cells (Harris et al., 2014). It has also been researched by Dr. Syed et al. that TNF- $\alpha$ upregulates the expression of TLR2, 8, and 9 (2007). To determine if the gene had been activated in each treatment, RT-qPCR was also performed to measure the gene expression of TNF- $\alpha$ (Figure 1B).

Previous research conducted by Dr. Ning and colleagues discovered that the expression of IRF-7 depends on the activation of TLR. Therefore, if the expression of IRF-7 is present, then at least one TLR must have been activated. Furthermore, in breast cancer, it has been shown that the promotion of the IRF-7 gene expression suppresses metastasis (Bidwell et al., 2012). When activated, this signaling pathway may have a beneficial effect on cancer. When BMDMs were treated with OPSS liposomes and curcumin, an increase in the gene expression of IRF-7 (Figure 1C) over control and PBS was observed, suggesting the treatment may have antitumor effects.

IP-10 is an additional gene involved in antitumor activity, as shown in a study done by Dr. Liu et al. (2011). Previous research conducted by Dr. An and colleagues have demonstrated that the expression of TLR additionally stimulates the expression of IP-10 (2007). Measuring IP-10 gene expression serves as an additional signal of TLR activation. The antitumor activity exhibited by IP-10 involves polarizing macrophages to be antitumoral, inducing apoptosis, and regulating cell growth and proliferation. The expression of the IP-10 gene was upregulated in BMDMs treated with OPSS and curcumin liposomes compared to control and PBS cells (Figure 1D). 

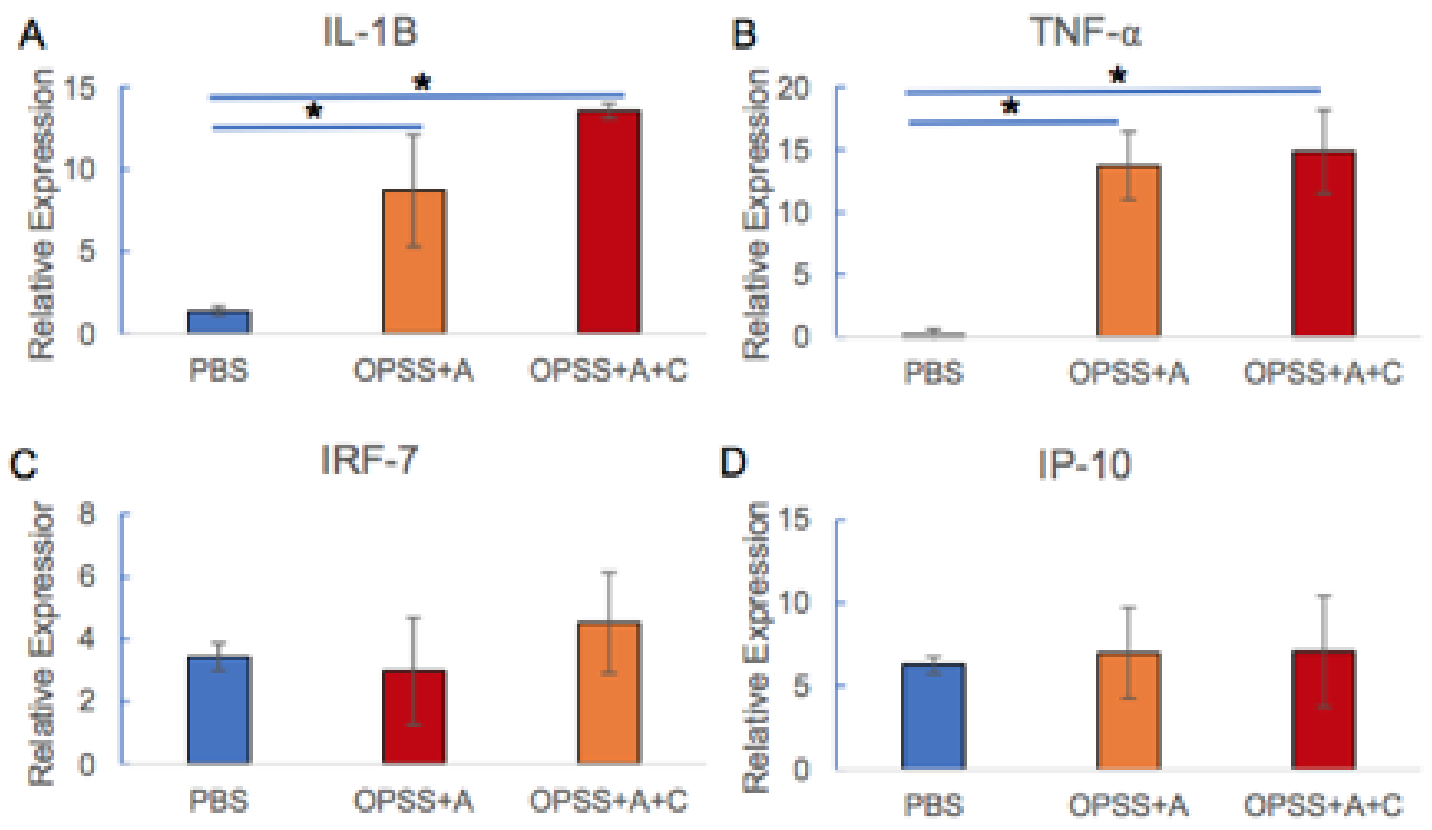

Figure 1. Gene expression relative to $\beta$-Actin expression in BMDM cells.

BMDM cells were treated with PBS, OPSS-liposomes encapsulating TLR agonists alone (OPSS+A) or with curcu$\min (\mathrm{OPSS}+\mathrm{A}+\mathrm{C})$ for 24 hours. RT-PCR was used to analyze the relative expression of A) IL-1B, B) TNF- $\alpha, \mathrm{C}$ ) IRF-7, and D) IP-10 mRNA gene expression in response to treatment. Data are expressed as mean \pm standard error $(\mathrm{n}=4)$ and normalized to B-Actin. ${ }^{*} \mathrm{p}$-value $<0.05$.

A

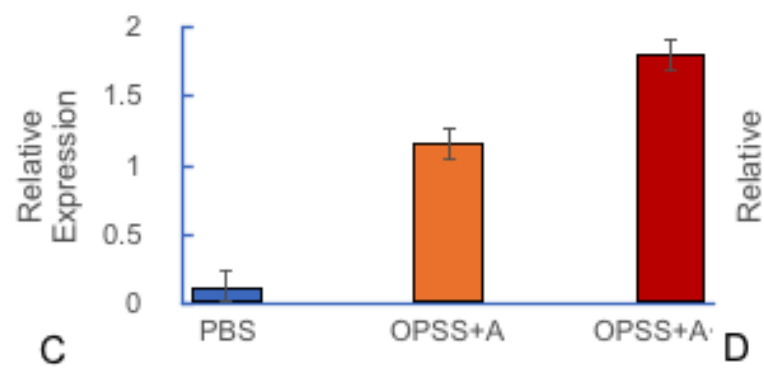

TLR8

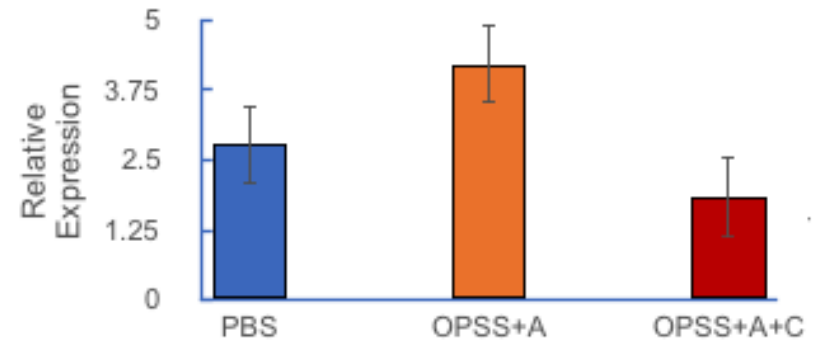

B

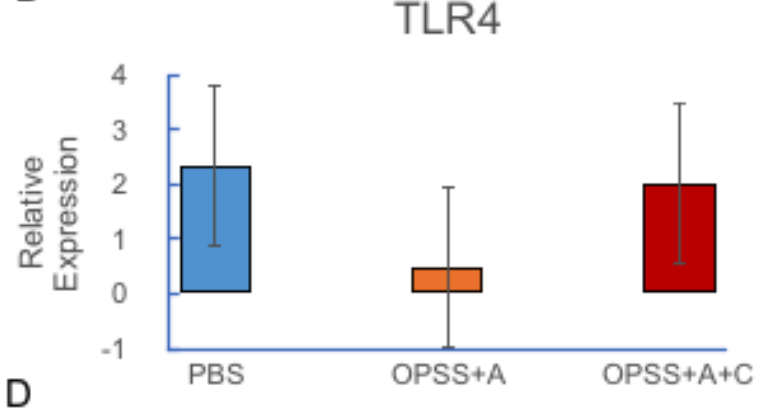

TLR9

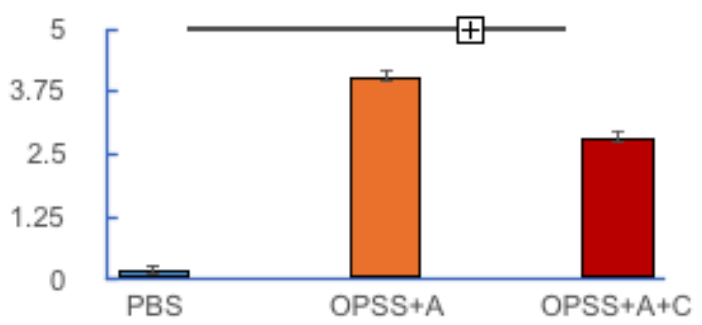

Figure 2. Toll-like receptor (TLR) gene expression relative to $\beta$-Actin expression. 
Murine bone marrow derived cells were treated with PBS, OPSS-liposomes encapsulating TLR agonists alone $(\mathrm{OPSS}+\mathrm{A})$ or with curcumin (OPSS $+\mathrm{A}+\mathrm{C})$ for 24 hours. RT-PCR was used to analyze the relative expression of A) TLR2 B) TLR4, C) TLR8, and D) TLR9 mRNA gene expression in response to treatment. Data are expressed as mean \pm standard error $(n=4)$ and normalized to B-Actin. *p-value $<0.05$.

\section{Flow Cytometry Results}

The percent positive expression of MHC-II, CD40, and CD83 was used to determine the activation of myeloid cells in vitro. These molecules have been shown to promote TLR triggered immune responses, which can help determine the extent to which the macrophages were activated (Liu et al., 2011). MHCII, CD40, and CD83 are immune-stimulating proteins usually found on antigen-presenting cells, such as macrophages, and can initiate an immune response. According to Dr. Ziduo et al., these proteins are markers for antigen-presenting cells that can measure the activation of the macrophages (2019). The graph in Figure 3 below shows that the OPSS liposome and TLR agonist treatment significantly activated BMDMs in vitro regardless of curcumin compared to PBS treated cells. Data are presented as mean \pm standard error. Differences among groups were determined using an unpaired two-tailed Student's t-test. Pvalues of less than 0.05 were considered significant and are indicated by an asterisk over the data or a bracket between samples.

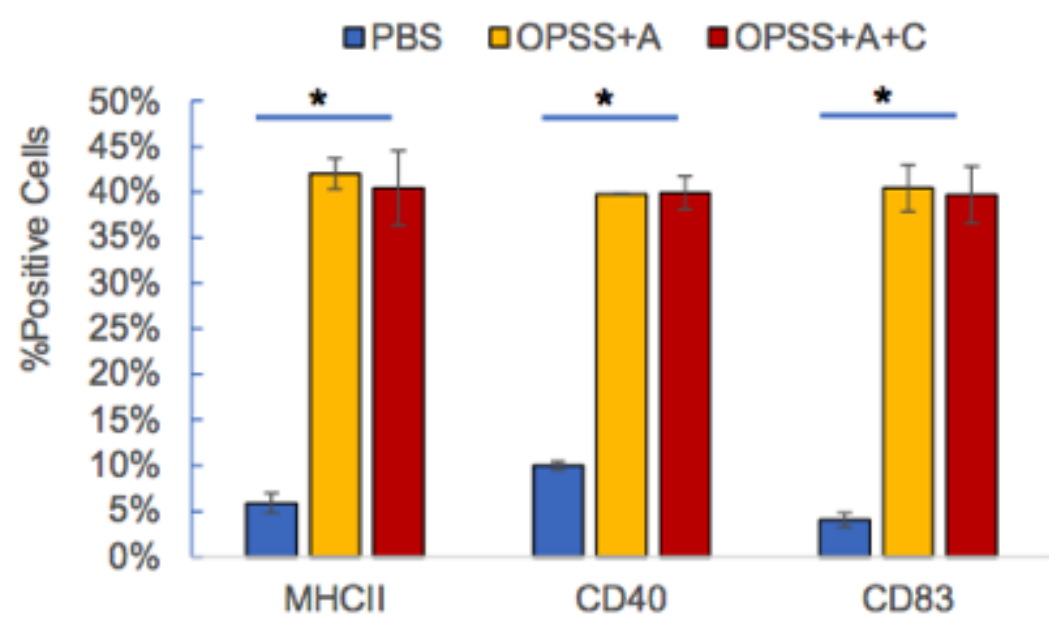

Figure 3. Percent Positive Expression of Immune-Stimulating Proteins in BMDMs.

Note: OPSS liposomes and TLR agonist treatments (OPSS+A) activate MHC-II, CD40, CD83 in BMDMs at the same level as the OPSS liposomes containing curcumin and TLR agonist (OPSS+A+C) $(n=3)$. $* p<0.001$

However, when BMDMs were co-cultured with 4T1 cancer cells, it can be observed that the OPSS liposome delivery of curcumin significantly induced MHC-II expression compared to all other conditions (Figure 4). 


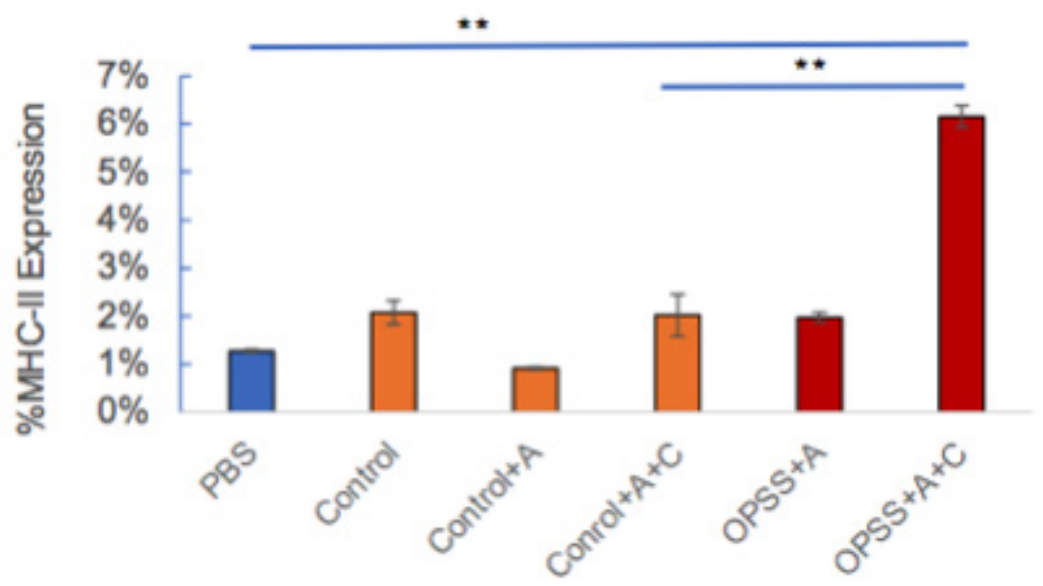

Figure 4. OPSS liposomes Containing Curcumin and TLR agonist upregulates MHC-II expression on BMDMs cocultured with cancer cells.

Note: The OPSS liposome, curcumin, and TLR agonist treatment (OPSS+A+C) displayed the highest level of MHCII expression in BMDMs co-cultured with 4T1 mammary carcinoma cells ( $\mathrm{n}=3) . * \mathrm{p}<0.001$

After an additional examination of the MHC-II positive cells treated with OPSS liposomes, curcumin, and TLR agonists, it was found that there was an increased expression of co-stimulatory markers CD40 and CD83. As mentioned above, CD40 and CD83 are co-stimulatory proteins that can activate an immune response. To determine if the macrophages were polarized into a non-tumor-associating form, the percent expression of the CD40 and CD83 molecules were measured in macrophages that were MHC-II positive and MHC-II negative. The increased expression of these proteins indicates the activation of the macrophages, which is beneficial to the immune response against breast cancer. A comparison of the percent expression of CD40 and CD83 in MHC-II positive and MHC-II negative cells can be seen in Figure 5.

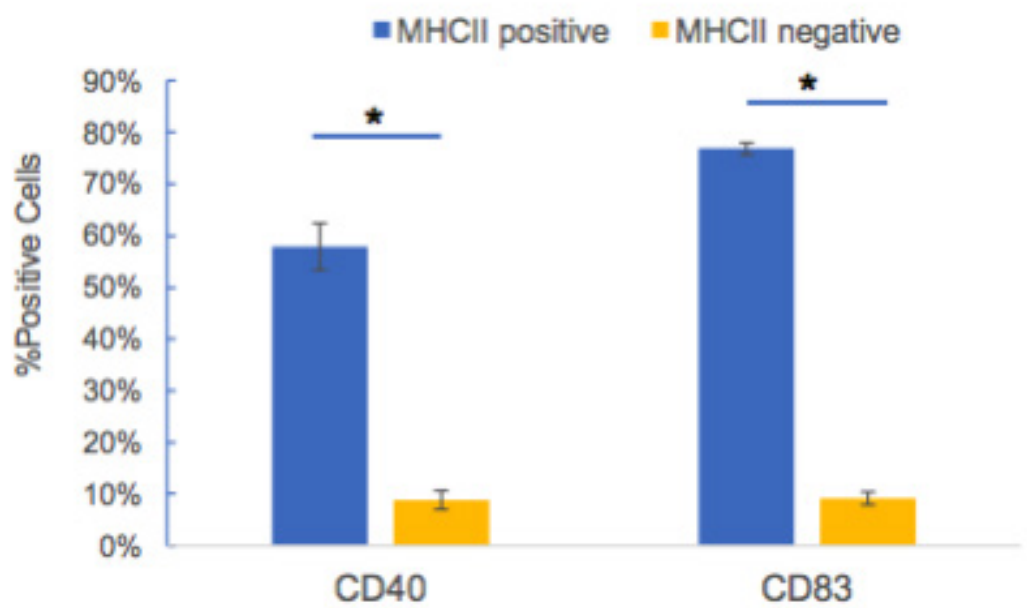

Figure 5. Percent Expression of CD40 and CD83 on OPSS Liposomes Curcumin treated MHC-II Positive and MHCII Negative BMDMs co-cultured with cancer cells.

Note: Percent expression of CD40 and CD83, co-stimulatory activation markers in MHC-II positive myeloid cells cocultured with 4T1 mammary carcinoma cells $(\mathrm{n}=3) .{ }^{*} \mathrm{p}<0.001$ 


\section{Discussion}

In this study, potential treatments to block the STAT3 signaling pathway were explored by delivering curcumin and TLR agonists using OPSS liposomes. The overall goal of the study was to block the STAT3 pathway to allow for the activation of the anti-tumoral form of macrophages. Previous research has recommended using liposomes as a delivery mechanism for curcumin to macrophages, but none had explored the use of OPSS liposomes to deliver curcumin and/or TLR agonists to macrophages.

The activation of the IL-1 $\beta$, TNF- $\alpha$, IRF-7, and IP-10 genes served as "signal genes" to affirm the expression of the TLR 2, 4, 8, and 9 genes. In all four "signal genes," the OPSS and curcumin treatment had the highest level of expression compared to the OPSS, curcumin, and TLR agonist treatment. Being a part of the IL-1/TLR receptor "superfamily," the activation of the IL- $1 \beta$ gene expression suggests OPSS liposome and curcumin treatment inhibited the STAT3 signaling pathway. However, further functional analysis should be considered to confirm STAT3 inhibition. In this context, it appears that the OPSS liposomes and curcumin treatment were the most successful in inhibiting the STAT3 signaling pathway. RT-qPCR determined the expression of genes, and the differing levels of gene expression signaled the inhibition of the STAT3 signaling pathway. A more specific method with fewer limitations would have been intracellular fluorescent indicators, which were not available when conducting this study. Future studies on this subject should consider more specific methods to indicate the inhibition of signaling pathways, such as intracellular fluorescent indicators.

After further examination of the expression of the TLR genes, the genes can be split into three groups depending on the relative level of activation: normal levels of gene expression, a decrease in gene expression, and an increase in gene expression. Despite belonging to the same receptor family, differences in expression in the presence of OPSS liposomes, curcumin, and TLR agonists may have arisen due to the variations in specificities of the TLR receptors. TLR gene expression analysis by RT-PCR indicated that BMDMs have variable expression regardless of treatment, indicated by the large error bars. The TLR2 gene displayed similar levels of expression among all three treatment groups, suggesting the treatments did not affect TLR2 gene expression. The TLR2 receptor encoded by the gene targets mainly Gram-positive bacteria, which suggests that OPSS liposomes, curcumin, and R848 (TLR7/8 agonist) do not activate the TLR2 receptor. A decrease in TLR4 and TLR8 gene expression was observed when BMDMs were treated with OPSS liposomes, regardless of the presence of curcumin. Previous studies conducted by Dr. Boozari et al. have presented that curcumin leads to the inhibition of TLR4 and TLR8 (2019). As suggested by Dr. Boozari et al., curcumin-mediated inhibition of a different pathway, such as the NF- $\mathrm{kB}$ signaling pathway, could have possibly led to the downregulation of TLR4 and TLR8 genes. The results of the aforementioned study and the results of this study suggest that OPSS liposomes on their own may inhibit other signaling pathways such as NF-kB. However, further research is needed to investigate these results. Of the four TLR genes measured, the TLR 9 gene exhibited a statistically significant activation of expression when treated with OPSS liposomes, curcumin, and TLR agonists. The expression of the TLR9 gene appeared to be stimulated by the presence of curcumin. Additionally, a cause of this stimulation may be due to the protein products of IL-1 $\beta$ and TNF- $\alpha$, which upregulates TLR9 moderately, which is consistent with other studies focusing upon TLR9 (Amcheslavsky et al., 2004). The aforementioned activation of IL$1 \beta$ and TNF- $\alpha$ may have played a role in TLR9 gene expression.

However, it is essential to remember that gene expression does not always result in protein expression, the products of which are crucial in activating macrophages for fighting cancer. Consequently, when defining the effectiveness of the treatment, it is vital to measure the expression of the protein product. However, the methodological choices when measuring protein expression were constrained to flow cytometry. Previous studies by Dr. Zhang et al. employed both Western Blot analysis and flow cytometry to measure protein expression, which is beneficial in confirming the validity of the results. For this study's purposes, flow cytometry was sufficient to measure the protein expression of cell surface markers. Future studies on this subject should consider employing a combination of Western Blot analysis and flow cytometry to measure protein expression accurately. 
The results after conducting flow cytometry measured the percent positive expression of cell surface markers MHC-II, CD40, and CD83. Of these three protein products, MHC-II is crucial in determining the activation of the immune response, particularly in macrophages. According to Dr. Brutkiewicz, STAT3 signaling inhibits the immune response, thereby inhibiting the expression of the MHC-II molecules on the surface of the macrophages (2017). Therefore, the expression of MHC-II is an indication of the inhibition of the STAT3 signaling pathway.

There were similar levels of MHC-II positive expression between the OPSS liposomes and TLR agonist treatment and the OPSS liposomes, TLR agonist, and curcumin treatment, suggesting that the curcumin may not have affected the MHC-II protein expression as it had on the gene expressions. However, to determine if the macrophages were polarized into an anti-tumor-associating form, the percent expression of the CD40 and CD83 molecules were measured in macrophages that were MHC-II positive and MHC-II negative. The increased expression of CD40 and CD83 molecules in MHC-II positive macrophages further confirms the polarization of macrophages from a tumorassociating form to an anti-tumor-associating form. These results are consistent with those of Dr. Zhang et al., whose study investigated STAT3 inhibition by using hydrazinocurcumin (2013). Although Dr. Zhang's study involved a synthetic analog of curcumin, the results of this study were consistent with those of the Dr.Zhang's study.

\section{Conclusion}

The results of this study point to the effectiveness of the OPSS liposomes in delivering curcumin and TLR agonist treatment, but further resesearch should be conducted on the effectiveness of the curcumin and TLR agonist treatment. Since STAT3 is a signaling pathway, and cannot be directly measured, it can only be measured by the protein expression, or the lack of protein expression. As Dr. Brutkiewicz has mentioned, STAT3 signaling inhibits the immune response, thereby inhibiting the expression of the MHC-II molecules on the surface of the macrophages, which means that MHC-II expression implies that the STAT3 pathway has been inhibited. The expression of MHC-II after the combination of curcumin and TLR agonists implies that the STAT3 signaling pathway has been inhibited, and the expression of TLR genes and other anti-tumor genes have been activated. Further research should test the combination of OPSS liposomes, curcumin, and TLR agonist treatment in an in vivo mouse model of cancer, which is more representative of the human immune response to treatments. Future studies using an in vivo mouse model should refer to methods set by Dr. Mukherjee and colleagues at the CUNY Graduate Center, who conducted a similar study focusing upon curcumin and glioblastoma (2016). However, these statements are based on RT-qPCR and flow cytometry results, and additional tests should be administered to further confirm STAT3 inhibition and TLR activation.

The replication of this experiment regarding the treatment combination of curcumin and TLR agonists can increase the scientific body of knowledge regarding the effect of these substances on macrophage activation. The implications of this experiment are broad due to the relative lack of knowledge about the utilization of OPSS liposomes, curcumin, and TLR agonists as a cancer immunotherapeutic treatment. The lack of information is significant since complement $\mathrm{C} 3$, the main component of the OPSS liposomes, is found in the blood of all humans, and can serve as an effective activator for the uptake of curcumin and TLR agonists, as well as the stimulation of macrophages.

\section{Acknowledgments}

I would like to express my sincere gratitude to my supervisor, WWAMI Prof. Holly Martinson, and her husband, WWAMI Prof. Max Kullberg for their persistent encouragement and inspiration during the course of study. Without their guidance, I wouldn't have been able to do any of this. I would also like to thank UAA undergraduate student Marisabel Ramirez and WWAMI medical student Keaton Rasic for providing instruction in various lab techniques and useful suggestions. I thank Mrs. Vani Pillai, Ms. Ellen Scott, and Mrs. Kaitlyn Williamson at Robert Service High School for their guidance and encouragement in writing a research paper. This work was supported by grants from the Institutional Development Award (IDeA) from the National Institute of General Medical Sciences of the National 
Institutes of Health under grant number P20GM103395, Alaska Run for Women, and the National Cancer Institute of the National Institutes of Health under grant number CA227740.

\section{References}

Akbharzadeh, A., Rezaei-Sadabady, R., Davaran, S., \& Joo, S. (2013). Liposome: Classification, preparation, and applications. Nanoscale Research Letters, 8(1).

Amcheslavsky, A., Zou, W., \& Bar-Shavit, Z. (2004). Toll-like receptor 9 regulates tumor necrosis factor- $\alpha$ expression by different mechanisms. Journal of Biological Chemistry. Retrieved from https://www.jbc.org/content/279/52/54039.full

Bidwell, B., Slaney, C., Withana, N., \& Forster, S. (2012). Silencing of Irf7 pathways in breast cancer cells promotes bone metastasis through immune escape. Nature Medicine. Retrieved from https://www.nature.com/articles/nm.2830

Boozari, M., Butler, A., \& Sahebkar, A. (2018). Impact of curcumin on toll-like receptors. Journal of Cellular Physiology, 12471-12482.

Brutkiewicz, R. (2017). Cell signaling pathways that regulate Ag presentation. Journal of Immunology. Retrieved from https:/www.ncbi.nlm.nih.gov/pmc/articles/PMC5119928/

Delneste, Y., Beauvillain, C., \& Jeannin, P. (2007). Immunité innée: Structure et fonction des TLR [Innate immunity: Structure and function of TLRs]. Med Sci (Paris). Abstract retrieved from https://www.ncbi.nlm.nih.gov/pub$\underline{\mathrm{med} / 17212934}$

DeNardo, D., \& Ruffell, B. (n.d.). Macrophages as regulators of tumor immunity and immunotherapy. Nature Reviews Immunology. https://doi.org/101038/s41577-019-0127-6

Feng, Y., Mu, R., Sing, P., Zhang, J., Dong, L., \& Wang, C. (2019). A toll-like receptor agonist mimicking microbial signal to generate tumor-suppressive macrophages. Nature Communications, (10). https://doi.org/10.1038/s41467019-10354-2

Francian, A., Namen, S., Stanley, M., Mann, K., Martinson, H., \& Kullberg, M. (2019). Intratumoral delivery of antigen with complement C3-bound liposomes reduces tumor growth in mice. Nanomedicine: Nanotechnology, Biology, and Medicine, 18, 326-335. https://doi.org/10.1016/j.nano.2018.10.009

Global, regional, and national age-sex specific mortality for 264 causes of death, 1980-2016: a systematic analysis for the Global Burden of Disease Study 2016. (n.d.). Global Health Metrics.

Harris, D., Bandyopadhyay, S., Maxwell, T., \& Willard, B. (2014). Tumor necrosis factor (TNF)- $\alpha$ induction of CXCL10 in endothelial cells requires protein arginine methyltransferase 5 (PRMT5)-mediated nuclear factor (NF)кB p65 methylation. Journal of Biological Chemistry. Retrieved from https://pubmed.ncbi.nlm.nih.gov/24753255/

Huyn, X., Chen, J., Wang, L., \& Ivashkiv, L. (2007). Crosstalk among Jak-STAT, toll-like receptor, and ITAMdependent pathways in macrophage activation. Journal of Leukocyte Biology. Retrieved from https://pubmed.ncbi.nlm.nih.gov/17502339/ 
Johnson, D., O'Keefe, R., \& Grandis, J. (2018). Targeting the IL-6/JAK/STAT3 signalling axis in cancer. Nature Reviews Clinical Oncology.

Kullberg, M., Martinson, H., Mann, K., \& Anchordoquy, T. (2015). Complement C3 mediated targeting of liposomes to granulocytic myeloid derived suppressor cells. Nanomedicine: Nanotechnology, Biology and Medicine, 11(6), 1355-1363.

Kunnumakkara, A., Anand, P., \& Aggarwal, B. (n.d.). Curcumin inhibits proliferation, invasion, angiogenesis and metastasis of different cancers through interaction with multiple cell signaling proteins. Cancer Letters, 199-225.

Kunnumakkara, A., Bordoloi, D., Choudhary, H., Banik, K., Gupta, S., \& Aggarwal, B. (2017). Curcumin mediates anticancer effects by modulating multiple cell signaling pathways. Portland Press, 1781-1799.

Ledesma, E., Martínez, I., Córdova, Y., Rodríguez-Sosa, M., Monroy, A., Mora, L., . . Osorio, E. (2004). Interleukin-1 Beta (IL-1beta) induces Tumor Necrosis Factor Alpha (TNF-alpha) expression on mouse myeloid multipotent cell line 32D cl3 and inhibits their proliferation. PubMed. Retrieved from https://pubmed.ncbi.nlm.nih.gov/15050606/

Liu, M., Guo, S., Jain, V., \& Singh, N. (2011). CXCL10/IP-10 in infectious diseases pathogenesis and potential therapeutic implications. Cytokine and Growth Factor Reviews. Retrieved from https://www.ncbi.nlm.nih.gov/pmc/articles/PMC3203691/\#S2title

Liu, M., Guo, S., \& Stiles, J. (2011). The emerging role of CXCL10 in cancer. Oncology Letters. Retrieved from https://www.ncbi.nlm.nih.gov/pmc/articles/PMC3406435/

Liu, X., Zhan, Z., Li, D., Xu, L., Zhang, P., Yao, H., \& Cao, X. (2011). Intracellular MHC Class II molecules promote TLR-triggered innate immune responses by maintaining activation of the kinase Btk. Immunology. Retrieved from https://pubmed.ncbi.nlm.nih.gov/21441935/

Lu, W.-D., Qin, Y., Yang, C., Li, L., \& Fu, Z.-X. (2013). Effect of curcumin on human colon cancer multidrug resistance in vitro and in vivo. Clinics (Sao Paulo). Retrieved from https://pubmed.ncbi.nlm.nih.gov/23778405/

Mukherjee, S., Baidoo, J., Fried, A., Atwi, D., Dolai, S., Boockvar, J., . . Banerjee, P. (2016). Curcumin changes the polarity of tumor-associated microglia and eliminates glioblastoma. International Journal of Cancer, $2838-2850$.

Murad, Y. M., Lyerly, H. K., \& Morse, M. A. (2007, August). CPG-7909 (PF-3512676, ProMune): Toll-like receptor-9 agonist in cancer therapy. Retrieved December 16, 2019, from https://www.ncbi.nlm.nih.gov/pub$\underline{\mathrm{med} / 17696823}$

Ning, S., Pagano, J., \& Barber, G. (2011). IRF7: Activation, regulation, modification and function. Genes and Immunity. Retrieved from https://www.ncbi.nlm.nih.gov/pmc/articles/PMC4437765/

O'Shea, J., Schwartz, D., Villarino, A., \& Gadina, M. (n.d.). The JAK-STAT pathway: Impact on human disease and therapeutic intervention. Annual Review of Medicine. Retrieved from https://www.ncbi.nlm.nih.gov/pmc/articles/PMC5634336/ 
Patrick, K., \& Stanbrook, M. (2018). Take turmeric with a grain of salt. CMAJ, 190(43). https://doi.org/10.1503/cmaj.181358

Sinjari, B., Pizzicannella, J., \& D'Aurora, M. (2019). Curcumin/Liposome nanotechnology as delivery platform for anti-inflammatory activities via NFkB/ERK/pERK pathway in human dental pulp treated with 2-HydroxyEthyl MethAcrylate (HEMA). Frontiers in Physiology. https://doi.org/10.3389/fphys.2019.00633

Sousa, S., Brion, R., Kronqvist, P., \& Sandholm, J. (n.d.). Human breast cancer cells educate macrophages toward the M2 activation status. Breast Cancer Research. Retrieved from https://www.ncbi.nlm.nih.gov/pubmed/26243145

Syed, M., Phulwani, N., \& Kielian, T. (2008). Tumor necrosis factor-alpha (TNF- $\alpha$ ) regulates toll-like receptor 2 (TLR2) expression in microglia. Journal of Neurochemistry. Retrieved from https://www.ncbi.nlm.nih.gov/pmc/articles/PMC2423670/

Varalakshmi, C., Ali, A., Pardhasaradhi, B., Srivastava, R., Singh, S., \& Khar, A. (2008). Immunomodulatory effects of curcumin: In-vivo. International Immunopharmacology, 8(5). https://doi.org/10.1016/j.intimp.2008.01.008

Villarino, A., Kanno, Y., \& O'Shea, J. (2015). Mechanisms of Jak/STAT signaling in immunity and disease. Journal of Immunology, 194(1). https://doi.org/10.4049/jimmunol.1401867

Zhang, W., Jiang, M., Chen, J., Zhang, R., Ye, Y., Liu, P., . . Yu, J. (2018). SOCS3 suppression promoted the recruitment of CD11b+Gr-1-F4/80-MHCII- early-stage myeloid-derived suppressor cells and accelerated interleukin6-related tumor invasion via affecting myeloid differentiation in breast cancer. Frontiers in Immunology, 9, 1-20. https://doi.org/10.3389/fimmu.2018.01699

Zhang, X., Tian, W., Wang, X., \& Dang, W. (2013). Hydrazinocurcumin encapsulated nanoparticles "re-educate" tumor-associated macrophages and exhibit anti-tumor effects on breast cancer following STAT3 suppression. PLOS One. Retrieved from https://journals.plos.org/plosone/article?id=10.1371/journal.pone.0065896 